\title{
M. Albour
}

\section{Régulation dynamique conflictuelle}

Revue française d'automatique, d'informatique et de recherche opérationnelle. Recherche opérationnelle, tome 4, no V3 (1970), p. 89-118.

<http://www.numdam.org/item?id=RO_1970_4_3_89_0>

(C) AFCET, 1970, tous droits réservés.

L'accès aux archives de la revue « Revue française d'automatique, d'informatique et de recherche opérationnelle. Recherche opérationnelle » implique l'accord avec les conditions générales d'utilisation (http://www.numdam.org/ legal.php). Toute utilisation commerciale ou impression systématique est constitutive d'une infraction pénale. Toute copie ou impression de ce fichier doit contenir la présente mention de copyright.

\section{Numdam}

Article numérisé dans le cadre du programme

Numérisation de documents anciens mathématiques

http://www.numdam.org/ 
R.I.R.O.

(4'e année, V-3, 1970, p. 89-118)

\title{
REGULATION DYNAMIQUE GONFLIGTUELLE
}

\author{
par M. Albouy (1)
}

\begin{abstract}
Résumé. - En étudiant les situations de conflit ou de coopération sous un point de vue strictement statique, la théorie mathématique des jeux ignore délibérément tous les problèmes liés à l'information et au déroulement du temps. Parallèlement, la théorie de la commande optimale suppose que l'organe de décision est seul avec la nature à agir sur l'évolution du système.

Il est donc indispensable, tant au niveau de la réflexion économique qu'au niveau de l'action d'analyser ce qui se passe lorsque plusieurs centres de commande agissent sur l'évolution du système. Cet article basé sur les travaux de plusieurs auteurs, notamment ceux de J. H. Case, Y. C. Ho, A. W. Starr, R. D. Behn, I. B. Rhodes et D. G. Luenberger, constitue donc une généralisation à la fois de la théorie des jeux et de la théorie de la commande.

De la première il retient les notions fondamentales : équilibre minimax, équilibre de Nash-Cournot, optimum de Pareto ; de la seconde, il conserve la formulation en terme de système ainsi que les techniques de séparation au moyen de l'Hamiltonien et des fonctions duales associées.

Ce dernier point de vue reste fondamental car il nous permet de donner une interprétation économique aux différentes variables utilisées.

La lecture de cet article devrait convaincre le lecteur qu'un grand nombre de conflits économiques peuvent être schématisés sous forme d'un jeu différentiel. On peut en attendre une meilleure compréhension du double-jeu d'alliance et de concurrence auquel se livrent les entreprises et, sur un plan plus théorique, une généralisation des modèles classiques de micro-économie.
\end{abstract}

La théorie mathématique des jeux se borne à étudier les situations de conflit ou de coopération sous un angle strictement statique. Ce faisant, elle ignore délibérément l'enchaînement temporel des décisions et tous les problèmes liés à l'information qu'il s'agisse de l'observation du système de la connaissance des autres joueurs ou de l'information sur la Nature. D'un autre côté, la théorie de la commande optimale suppose que l'organe de décision est seul, avec la Nature, à agir sur l'évolution du système.

(1) Etudes Economiques générales d'E.D.F.

Maître de conférences à l'École Polytechnique. 
Il est donc indispensable, tant au niveau de la réflexion économique qu'au niveau de l'action, d'analyser ce qui se passe lorsque plusieurs centres de commande peuvent agir sur l'évolution d'un système, c'est-àdire quand le problème d'interdépendance dans l'espace se double d'un problème d'interdépendance dans le temps.

Cet article constitue donc une généralisation à la fois de la théorie des jeux et de la théorie de la commande. De la première il retient les notions fondamentales : équilibre minimax, équilibre de Nash-Cournot, optimum de Pareto ; de la seconde il conserve la formalisation en termes de systèmes ainsi que les techniques de séparation au moyen de l'Hamiltonien et des fonctions duales associées. Ce dernier point de vue reste fondamental car il permet de donner une interprétation économique aux différentes variables duales utilisées. En effet, depuis les premiers travaux d'Isaacs [1] sur les jeux du type poursuite-évasion, la théorie des jeux différentiels paraît s'orienter de plus en plus vers la modélisation des conflits économiques en s'efforçant dans le même temps de mettre au point les techniques de calcul correspondantes. Cette orientation est le fait des travaux de plusieurs auteurs cités en référence, notamment ceux de J. H. Case, Y. C. Ho, A. W. Starr, R. D. Behn, I. B. Rhodes et D. G. Luenberger.

Nous nous bornerons dans cet article à une présentation rapide composée de trois parties:

- un exposé général sur la théorie des jeux différentiels à $N$ personnes et sur son interprétation économique,

- l'application de cette théorie à la solution d'un jeu "linéairequadratique " (évolution linéaire et fonctions économiques quadratiques),

- enfin l'analyse de quelques schémas particuliers (duel, jeu à deux personnes, organisation décomposable, organisation tendue vers un même but, organisation hiérarchisée) assortie d'un certain nombre d'exemples.

\section{EXPOSE GENERAL SUR LA THEORIE MATHEMATIQUE DES JEUX DIFFERENTIELS}

Considérons un système dont l'évolution est repérée à tout instant $t \in(0, T)$ par un vecteur $x(t)$ à $n$ composantes. Nous supposerons que cette évolution peut être représentée par un système d'équations différentielles :

$$
\frac{\mathrm{d} x}{\mathrm{~d} t}=x=f\left(x, t, u_{1}, \ldots u_{i}, u_{N}\right)
$$

équations dans lesquelles les vecteurs $u_{1}, \ldots, u_{i}, u_{N}$ désignent les décisions des différents joueurs tandis que la variable $t$ mesure l'effet des "paramètres naturels ". L'état du système à l'instant initial, $x(0)$, est supposé connu $x(0)=x_{0}$. 
La structure d'évaluation du joueur $i$ peut s'écrire de deux façons différentes :

ou bien $\quad \operatorname{Max} \int_{0}^{T} L_{i}\left(x, t, u_{1}, \ldots u_{i}, u_{N}\right) \mathrm{d} t+\Phi_{i}(x(T), T)$

la fonction $\Phi_{i}$ représentant la valeur que le joueur $(i)$ attribue à l'instant $T$ au système lorsque celui-ci se trouve dans l'état $x(T)$

ou bien

$$
\operatorname{Max} \int_{0}^{T} L_{i}\left(x, t, u_{1}, \ldots u_{i}, u_{N}\right) \mathrm{d} t
$$

sous les contraintes $g_{i}(x(T), T) \geqslant 0$

l'inégalité vectorielle $g_{i}(x(T), T) \geqslant 0$ représentant la cible que le joueur (i) se propose d'atteindre à l'époque $T$.

L'évolution du système est générale ment limitée par un certain nombre de contraintes qui portent soit sur l'état du système, soit sur les décisions des différents joueurs, soit encore sur les deux à la fois. Nous nous bornerons par la suite à analyser deux cas particuliers :

- le cas où il n'existe aucune contrainte

- le cas où le domaine des décisions possibles du joueur ( $i$ ) dépend uniquement de l'époque $t$ et de l'état du système à cette époque :

$$
g_{i}\left(x, u_{i}, t\right) \geqslant 0 .
$$

En ce qui concerne le réseau d'information, nous admettrons que chaque joueur possède une connaissance parfaite des termes du conflit et des forces en présence; plus précisément, il connaît les équations qui régissent l'évolution du système, la forme des contraintes et les différentes structures d'évaluation.

Il n'en est pas de même de l'observation du système :

- ou bien on suppose que les joueurs ont les moyens d'observer continûment et parfaitement la trajectoire; dans ce cas le système sera géré en boucle fermée c'est-à-dire que chaque joueur définira pour toute époque $t \in(0, T)$ une stratégie décisionnelle du type $u[x(t), t]$.

- ou bien on suppose que les joueurs ne disposent d'aucune information sur l'évolution du vecteur $x(t)$; dans ce cas, le système sera géré en boucle ouverte, c'est-à-dire que chaque joueur définira pour chaque époque $t \in(0, T)$ une stratégie décisionnelle du type $u(t)$.

\subsection{Trajectoires Minimax}

Supposons tout d'abord que chacun des centres de décision, pour des raisons psychologiques ou par manque d'information perçoive le conflit sous forme d'un duel entre lui-même et les autres joueurs. 


\subsubsection{Conditions d'équilibre minimax en boucle ouverte}

En l'absence de contraintes et de cibles sur l'état final, la stratégie de défense $\bar{u}_{i}(t)$ en boucle ouverte du joueur (i) face à la coalition présumée des autres joueurs $j \neq(i)$ consiste à résoudre le problème :

$$
\left.\underset{u i}{\operatorname{Max}}\left[\underset{\left(u_{i}, j \neq i\right)}{\operatorname{Min}}\left[\int_{0}^{T} L_{i}\left(x, u_{i}, u_{j}, t\right) \mathrm{d} t+\Phi_{i}\left[x_{(} t\right), T\right]\right]\right]
$$

avec $\stackrel{\circ}{x}=f\left(x, u_{i}, u_{j}, t\right)$ et $x(0)=x_{0}$

le terme $\left(u_{j}, j \neq i\right)$ désignant l'ensemble des décisions de la coalition.

En formant l'Hamiltonien $\mathfrak{H}_{i}=L_{i}+\psi_{i} \cdot f\left(x, u_{i}, u_{j}, t\right)$ on peut décomposer ce duel dynamique en une suite de duels statiques :

$$
\underset{u_{i}}{\operatorname{Max}}\left[\underset{\left(u_{i}, j \neq i\right)}{\operatorname{Min}}\left[\mathscr{H}_{i}\right]\right]
$$

liés par les équations d'évolution :

$$
\begin{array}{ll}
\stackrel{\circ}{x}=f\left(x, u_{i}, u_{j}, t\right) ; & x(0)=x_{0} \\
\dot{\leftrightarrow}_{i}=-\nabla_{x}\left(\mathcal{H}_{i}\right) ; & \psi_{i}(T)=\nabla\left(\Phi_{i}\right)
\end{array}
$$

On constate que les composantes du vecteur $x$ qui n'apparaissent pas dans la fonction d'évaluation du joueur (i) n'interviennent pas dans l'Hamiltonien $\mathfrak{H}_{i}$ de ce joueur : en effet dans ce cas les fonctions duales associées à ces composantes sont telles que $\psi_{i}(T)=0$ et $\dot{\psi}_{i}=0$, soit $\psi_{i}(t)=0 \quad \forall t \in(0, T)$.

Dans ce cas la stratégie de défense $\bar{u}_{i}(t)$ du centre $(i)$ et la stratégie de menace $\hat{u}_{j}$ des autres joueurs formeront localement un col

si

$$
\begin{aligned}
& \text { (a) } \stackrel{\stackrel{\circ}{x}}{=}=f\left(x, u_{i}, u_{j}, t\right), \quad x(0)=x_{0} \\
& \dot{\leftrightarrow}_{i}=-\nabla\left[\mathcal{H}_{i}\right]=-\frac{\partial \mathcal{H}_{i}}{\partial x} ; \quad \psi_{i}(T)=\nabla\left[\Phi_{i}\right] \\
& 0=\nabla_{u_{i}}\left[\mathscr{H}_{i}\right]\left(x, t, \bar{u}_{i}, \hat{u}_{j}, \psi_{i}\right) \\
& 0=\nabla_{\left(u_{j}, j \neq i\right)}\left[\mathcal{H}_{i}\left(x, t, \bar{u}_{i}, \hat{u}_{j}, \psi_{i}\right)\right]
\end{aligned}
$$

(b) |la matrice $\nabla u_{i} u_{i}\left[\mathscr{H}_{i}\right]$ est définie négative et la matrice $\nabla u_{j} u_{j}\left[\mathcal{H}_{i}\right]$ définie positive

Bien entendu, si l'Hamiltonien $\mathscr{H}_{i}$ est une fonction concave en $u_{i}$ et convexe en $u_{j}$, les conditions $(a)$ sont suffisantes pour caractériser le minimum absolu de $\mathfrak{H}_{i}$ par rapport à $u_{j}$ et son maximum absolu par rapport à $u_{i}$.

Ayant obtenu la stratégie de défense $u_{i}$ du centre $(i)$, si l'on veut définir la trajectoire minimax, il faut recommencer le calcul pour tous les autres joueurs. Le calcul de la trajectoire minimax impose donc de résoudre $N$ jeux dynamiques à deux personnes à somme nulle. 
Bien entendu, si tous les joueurs adoptent leur stratégie de défense ou stratégie minimax la trajectoire résultante ne coïncide pas avec les trajectoires prévues par chacun des joueurs. Pour qu'elles coïncident, il faudrait que le conflit se réduise effectivement à un duel.

En présence de cibles (du type $g_{i}[x(T), T] \geqslant 0$ ) et de contraintes (du type $g_{i}\left(x, u_{i}, t\right) \geqslant 0$ ) la méthode reste inchangée, à condition toutefois que le système demeure contrôlable. On démontre en effet que lorsqu'elle existe, la situation d'équilibre entre le joueur $(i)$ et la coalition présumée des autres joueurs satisfait aux conditions du premier ordre :

$$
\begin{aligned}
& \dot{\psi}_{i}(t)=-\nabla_{x}\left[\mathcal{H}_{i}^{c}\left(x, \bar{u}_{i}, \hat{u}_{j}, t, \psi_{i}\right)\right]=-\frac{\partial \mathcal{H}_{i}^{c}}{\partial x} \\
& \psi_{i}(T)=\sum_{k=1}^{N} \lambda_{k}(t) \cdot \nabla\left[g_{k}[x(T), T]\right] \\
& \text { avec } \lambda_{k}(t) \cdot g_{k}[x(T), T]=0 \quad \forall k=1 \ldots N \\
& \stackrel{\grave{x}}{=}=f\left(x, \bar{u}_{i}, \hat{u}_{j}, t\right) ; \quad x(0)=x_{0} \\
& \mathscr{H}_{i}^{c}=L_{i}+\psi_{i} \cdot f\left(x, u_{i}, u_{j}, t\right)+\sum_{k=1}^{N} \lambda_{k}(t) \cdot g_{k}\left(x, u_{k}, t\right) \\
& \text { avec } \quad \lambda_{i}(t) \cdot g_{i}\left(x, \bar{u}_{i}, t\right)=0 \\
& \begin{array}{c}
\lambda_{j}(t) \cdot g_{j}\left(x, \hat{u}_{j}, t\right)=0, \text { pour } \quad(j \neq i, j=1 \ldots N) \\
\nabla_{u_{i}}\left[\mathcal{H}_{i}^{c}\right]=0 ; \quad \nabla_{\left(u_{j}, j\right.} \#{ }_{i)}\left[\mathfrak{H}_{i}^{c}\right]=0
\end{array}
\end{aligned}
$$

Par ailleurs, on démontre que ces conditions sont suffisantes si l'Hamiltonien complété $\mathfrak{H}_{i}^{c}$ est une fonction concave par rapport à $u_{i}$ et convexe par rapport à $u_{j}$. Bien que la présence de contraintes rende le calcul plus difficile, ces équations permettent théoriquement de définir la stratégie de défense $\bar{u}_{i}(t)$ du joueur $(i)$. Pour déterminer la trajectoire minimax il reste ensuite à résoudre $N$ duels du même type.

\subsubsection{Conditions d'équilibre minimax en boucle fermée $\bar{u}_{i}[x(t), t]$}

Si l'on admet que tous les centres de décision sont informés à chaque instant de l'état du système, la stratégie de défense qu'ils adopteront tiendra compte des réactions de leurs adversaires.

Cet effet de réaction se concrétise théoriquement par une évolution différente des fonctions duales $\psi_{\imath}(t)$.

En l'absence de contraintes, les conditions énoncées au paragraphe précédent restent inchangées à l'exception de l'équation de $\stackrel{\circ}{\psi}_{i}$ qui s'écrit :

$$
\dot{\Psi}_{i}=-\frac{\partial \mathcal{F}_{i}}{\partial x}-\sum_{k=1}^{N} \frac{\partial \mathcal{F}_{i}}{\partial u_{k}} \cdot \frac{\partial u_{k}}{\partial x}
$$


Mais comme par ailleurs, à l'optimum :

$$
\frac{\partial \mathcal{H E}_{i}}{\partial u_{i}}=-\frac{\partial \mathcal{H E}_{i}}{\partial_{\left(u_{i}, j \neq i\right)}}=0
$$

l'effet de réaction s'annule.

La stratégie minimax en boucle ouserte coïncide donc avec la stratégie minimax en boucle fermée.

Le lecteur pourra vérifier facilement que lorsqu'il existe des contraintes du type mentionné ci-dessus, la stratégie minimax en boucle ouverte se confond encore a vec la stratégie minimax en boucle fermée. Il convient toutefois de noter qu'il est peu probable que des joueurs parfaitement informés de l'état du système maintiennent leur stratégie minimax. Ils s'apercevront en effet immédiatement que la trajectoire obtenue est plus favorable que la trajectoire prévue : aussi, à moins d'un blocage psychologique ou d'une absence totale d'information sur les intentions de leurs rivaux, ils seront tentés d'abandonner leur attitude défensive.

\subsection{Trajectoires d'équilibre de Nash-Cournot}

Une attitude plus réaliste consiste à rechercher une situation de compromis. On dit que le système suit une trajectoire d'équilibre au sens de Nash-Cournot si, à partir de cette trajectoire, aucun des joueurs ne peut améliorer son résultat en modifiant unilatéralement sa stratégie.

Mathématiquement la stratégie $\left[u_{1}^{*}, \ldots u_{i}^{*}, u_{N}^{*}\right]$ est une stratégie d'équilibre si $\forall i=1 \ldots N$ :

$$
\begin{aligned}
& \int_{0}^{T} L_{i}\left(x, u_{1}^{*}, u_{i}^{*}, u_{N}^{*}, t\right) \mathrm{d} t \geqslant \int_{0}^{T} L_{i}\left(x, u_{1}^{*}, u_{i}, u_{N}^{*}, t\right) \mathrm{d} t \\
& +\Phi_{i}\left[{ }_{x}^{*}(T), T\right] \\
& +\Phi_{i}[x(T), T]
\end{aligned}
$$

Il est évident que si le joueur (i) connaissait les stratégies de NashCournot de ses rivaux, il lui suffirait pour définir sa stratégie $u_{i}^{*}$ de résoudre un problème classique de commande optimale. Comme les stratégies des autres joueurs sont inconnues, il lui faut résoudre un problème de commande optimale paramétré. On calcule ainsi l'ensemble des stratégies de meilleure réponse de chaque joueur face aux décisions possibles de ses rivaux et c'est l'intersection des ensembles de meilleure réponse qui définit les stratégies d'équilibre. Lorsque cet ensemble est vide, il n'existe aucune trajectoire d'équilibre. Lorsque cet ensemble est formé de plusieurs éléments, on obtient un faisceau de trajectoires d'équilibre.

Dans l'exposé qui suit, nous distinguerons, comme précédemment, deux types de stratégies :

- les stratégies en boucle ouverte,

— les stratégies en boucle fermée. 


\subsubsection{Conditions d'équilibre de Nash-Cournot en boucle ouverte}

Pour écrire les conditions caractéristiques de l'équilibre, on considère tour à tour chaque joueur et on suppose que celui-ci connaît les stratégies d'équilibre de Nash-Cournot en boucle ouverte de tous ses rivaux, c'est-à-dire l'ensemble des fonctions $u_{j}^{*}(t)$ pour $(j \neq i, j=1 \ldots N)$. Dans ce cas, les théorèmes classiques de la théorie de la commande optima lenous permettent d'écrire les conditions auxquelles doit satisfaire la commande $u_{i}^{*}(t)$ pour que le joueur $(i)$ réalise localement ou globalement le résultat le plus favorable. En généralisant ces conditions aux $N$ joueurs, on obtient les conditions caractéristiques d'un équilibre local ou global.

En l'absence de contraintes, ces conditions sont relativement simples. En effet, comme l'Hamiltonien d'un joueur quelconque $(i)$ s'écrit :

$$
\mathscr{H}_{i}=L_{i}\left(x, t, u_{1}^{*}, \ldots u_{i}, u_{N}^{*}\right)+\psi_{i} \cdot f\left(x, t, u_{1}^{*}, \ldots u_{i}, \ldots u_{N}^{*}\right)
$$

les conditions caractéristiques d'un équilibre local sont les suivantes :

a)

$$
\begin{aligned}
\stackrel{\circ}{x} & =f\left(x, t, u_{1}^{*}, \ldots u_{i}^{*} \ldots u_{N}^{*}\right) ; x(0)=x_{0} \\
\stackrel{\circ}{\psi}_{i}(t) & =-\nabla_{x}\left[\mathscr{H}_{i}\right]=-\frac{\partial \mathcal{H}_{i}}{\partial x}, \quad \psi_{i}(T)=\nabla\left[\Phi_{i}\right]=\frac{\partial \Phi_{i}[x(,), T]}{\partial x(T)} \\
\forall i & =1 \ldots N \\
0 & =\nabla\left[\mathcal{H}_{i}\right]=\frac{\partial \mathscr{H}_{i}}{\partial u_{i}} ; \quad \forall i=1 \ldots N
\end{aligned}
$$

(b) La matrice $\nabla u_{i} u_{i}\left[\mathcal{H}_{i}\right]$ est définie négative le long de la trajectoire d'équilibre pour tous les joueurs $i=1 \ldots N$.

Par ailleurs, si le long de la trajectoire d'équilibre les différents Hamiltoniens $\mathfrak{H}_{i}$ sont des fonctions concaves de $u_{i}$, on sait que les conditions (a) suffisent à caractériser l'équilibre global ou absolu du système.

En présence de contraintes, sous réserve que le système soit contrôlable, on définit pour chaque joueur $(i)$ un Hamiltonien complété :

$$
\begin{aligned}
\mathscr{H}_{i}^{c}=L_{i}\left(x, t, u_{1}^{*}, \ldots u_{i}, \ldots u_{N}^{*}\right)+\psi_{i} \cdot f\left(x, t, u_{1}^{*}, \ldots u_{i}, \ldots u_{N}^{*}\right) & \\
& +\lambda_{i}(t) \cdot g_{i}\left(x, u_{i}, t\right)
\end{aligned}
$$

On utilise ensuite ces Hamiltoniens $\mathfrak{H}_{i}^{c}$ en lieu et place des Hamiltoniens précédents pour exprimer, lorsque celles-ci existent, les conditions caractéristiques des trajectoires d'équilibre. Bien entendu, il faut écrire certaines relations supplémentaires pour définir les nouvelles variables duales $\lambda_{i}(t)$, mais ces relations sont données par la maximisation de l'Hamiltonien $\mathcal{H}_{i}^{c}$ par rapport aux variables de décision $u_{i}$ sous les con- 
traintes $g_{i}\left(x, u_{i}, t\right) \geqslant 0$. On obtient ainsi les conditions nécessaires du premier ordre :

$$
\begin{aligned}
& \stackrel{\circ}{\Psi}_{i}=-\nabla_{x}\left[\mathcal{H}_{i}^{c}\right]=-\frac{\partial \mathscr{H}_{i}^{c}}{\partial x} ; \quad \psi_{i}(T)=\lambda_{i}(T) \cdot \nabla_{x}\left[g_{i}[x(T), T]\right] \\
& \stackrel{o}{x}=f\left(x, t, u_{1}^{*}, u_{i}^{*}, u_{N}^{*}\right) ; \quad x(0)=x_{0} \\
& \lambda_{i}(T) \cdot g_{i}\left[x^{*}(T), T\right]=0 \quad \text { (condition d'impact sur la cible) } \\
& \nabla\left[\mathscr{H}_{i}^{c}\right]=0 \\
& \lambda_{i}(t) \cdot g_{i}\left(x^{*} u_{i}^{*}, t\right)=0 \quad(\forall i=1 \ldots N)
\end{aligned}
$$

On sait par ailleurs que ces conditions sont suffisantes, si le long de la trajectoire d'équilibre, les Hamiltoniens complétés $\mathcal{H}_{i}^{c}$ sont des fonctions concaves par rapport à $u_{i}$.

\subsubsection{Conditions d'équilibre en boucle fermée}

On procède ici de manière analogue, en tenant compte des réactions des différents joueurs aux variations du vecteur " état ".

En l'absence de contraintes, on définit pour chaque joueur un Hamiltonien

$$
\mathscr{H}_{i}=L_{i}\left(x, t, u_{1}^{*}, u_{i}, u_{N}^{*}\right)+\psi_{i} \cdot f\left(x, t, u_{1}^{*} u_{i}, u_{N}^{*}\right)
$$

ce qui permet immédiatement d'écrire les conditions nécessaires et suffisantes d'un équilibre local :

$$
\begin{aligned}
\stackrel{\circ}{\psi}_{i}(t) & =-\frac{\partial \mathcal{H}_{i}}{\partial x}-\sum_{j=1} \frac{\partial \mathcal{H}_{i}}{\partial u_{j}^{*}} \cdot \frac{\partial u_{j}^{*}(x, t)}{\partial x} ; \psi_{i}(T)=\nabla_{x}\left[\Phi_{i}[x(T), T]\right] \\
\stackrel{\circ}{x} & =f\left(x, t, u_{1}^{*} \ldots u_{i}^{*}, \ldots u_{N}^{*}\right) ; \quad x(0)=x_{0} \\
0 & =\frac{\partial \mathcal{H}_{i}}{\partial u_{i}}\left(x, t, u_{1}^{*}, \ldots u_{N}^{*}\right) ; \quad \forall i=1 \ldots N
\end{aligned}
$$

b) La matrice des dérivées secondes $\nabla u_{i} u_{i}\left[\mathcal{H}_{i}\right]$ doit être définie négative le long de la trajectoire, quel que soit le joueur (i) considéré.

Bien entendu, si les fonctions $\mathcal{J}_{i}$, le long de la trajectoire, sont concaves (par rapport à $u_{i}$ ) les conditions $(a)$ suffisent à caractériser l'équilibre global ou absolu du système. De même, il n'y aurait aucune difficulté à écrire les conditions d'équilibre en présence de contraintes en utilisant les Hamiltoniens complétés $\mathcal{H}_{i}^{c}$.

Mais qu'il existe ou non des contraintes, un fait essentiel demeure : l'évolution des fonctions duales $\psi_{i}(t)$ dépend de la manière dont le joueur $(i)$ perçoit les réactions de ses adversaires aux variations de la trajectoire. Ce phénomène ne doit pas nous surprendre puisque toute régulation en boucle fermée engendre nécessairement entre les organes de commande un 
effet de couplage positif ou négatif qui apparaît sous forme d'un terme supplémentaire :

$$
\sum_{j=1}^{N} \frac{\partial \mathcal{H}_{i}}{\partial u_{j}} \cdot \frac{\partial u_{j}^{*}(x, t)}{\partial x}
$$

Ce terme ne devient nul que dans trois circonstances :

- dans le cas d'un centre de commande unique (absence de couplage $\left.\frac{\partial \mathcal{H}_{i}}{\partial u_{i}}=0\right)$,

- dans le cas d'un duel (annulation des effets de couplage $\left.\frac{\partial \mathscr{H}_{i}}{\partial u_{j}}=\frac{\partial \mathcal{H}_{i}}{\partial u_{i}}=0\right)$

- dans les régulations en boucle ouverte par absence de retour d'information.

Les conséquences de ce terme de couplage sont doubles :

$1^{\circ}$ La trajectoire d'équilibre de Nash en boucle ouverte ne coïncide pas avec la trajectoire d'équilibre de Nash en boucle fermée. Elles ne peuvent se confondre que si le conflit se réduit à un duel et dans ce cas, elles coïncident avec la trajectoire minimax.

$2^{\circ}$ Le calcul de la trajectoire d'équilibre en boucle fermée pose de sérieuses difficultés. En effet, dans l'hypothèse d'un centre de commande unique comme la trajectoire optimale en boucle ouverte se confond avec la trajectoire optimale en boucle fermée, on peut calculer cette trajectoire en cherchant la commande optimale $u(t)$ à partir de chaque situation initiale $(x, t)$. Par ailleurs, les équations d'évolution en $x$ et $\psi$ sont des équations différentielles ordinaires avec condition initiale sur $x$ et conditions finales sur $\psi$. Dans l'hypothèse d'une régulation conflictuelle en boucle fermée mettant en présence plusieurs centres de décision, les conditions d'équilibre s'expriment au contraire sous la forme d'un ensemble d'équations aux dérivées partielles, qu'il est beaucoup plus malaisé de résoudre.

Pour tourner la difficulté, certains auteurs ont imaginé d'abandonner l'approche variationnelle et de poser le problème en termes de " Programmation Dynamique ", c'est-à-dire d'utiliser une séparation au moyen de la fonction d'évaluation. Nash

A cet effet désignons par $u^{\star}(x, t)$ le $n$-uple de stratégies d'équilibre de et par

$$
\left[u_{1}(x, t), \ldots u_{i}(x, t) \ldots u_{N}(x, t)\right]
$$

$$
J_{i}^{*}(x, t)=\int_{t}^{T} L_{i}(x, u, t)+\mathrm{d} t+\Phi_{i}[x(T), T]
$$

la valeur de la fonction économique du joueur (i) à l'équilibre. Si les commandes $u_{i}(t)$ sont continues par morceaux, on sait que les fonctions de valeur $J_{i}^{*}$ sont elles-mêmes différentiables par morceaux. En appliquant 
le principe d'optimalité, on montre alors que les fonctions $J_{i}^{*}(x, t)$ sont solutions du système d'équations aux dérivées partielles

$$
\frac{\partial J_{i}^{*}}{\partial t}=-\underset{u_{i}}{\operatorname{Max}} \mathscr{H}_{i}\left[x, t, u_{1}^{*}, \ldots u_{i}, \ldots u_{N}^{*}, \frac{\partial J_{i}}{\partial x}\right]
$$

avec : $J_{i}[x(T), T]=\Phi_{i}[x(T), T] \quad, \quad \forall i=1 \ldots N$.

Ce système d'équation, qui suppose le principe d'optimalité, n'est rien d'autre que l'équation d'Hamilton-Jacobi-Bellman généralisée et la stratégie d'équilibre $u_{i}^{*}$ est celle qui réalise le maximum de l'Hamiltonien $\mathcal{H}_{i}$.

Le problème consiste donc à trouver pour chaque situation initiale $(x, t)$ le col du vecteur Hamiltonien $\left[\mathcal{H}_{1} \ldots \mathcal{H}_{i}, \mathcal{H}_{N}\right]$ c'est-à-dire le point d'équilibre du jeu statique à $N$ joueurs, puis à intégrer l'équation, d'Hamilton-Jacobi- étape par étape en remontant le cours du temps. On obtient ainsi, point par point, la trajectoire d'équilibre mais ce calcul n'est pas toujours possible. Il faut en effet qu'il existe pour chaque conflit statique un point d'équilibre unique et que la commande correspondante $u_{i}^{*}$ soit une fonction explicite de :

$$
\left[x, t, \frac{\partial J_{1}}{\partial x} \cdots \frac{\partial J_{N}}{\partial x}\right]
$$

Il faut aussi que l'on puisse, à partir des conditions finales, intégrer les équations

$$
\mid \begin{aligned}
\frac{\partial J_{i}}{\partial t} & =-\mathcal{H}_{i}\left[x, t, u_{1}^{*}, \ldots u_{N}^{*}, \frac{\partial J_{i}}{\partial x}\right] \\
\stackrel{\bullet}{x} & =f\left(x, u_{1}, u_{i}, \ldots u_{N}, t\right) .
\end{aligned}
$$

Ceci étant, les expériences ne sont pas encore assez nombreuses pour que l'on puisse d'ores et déjà donner la préférence à l'une ou l'autre des deux méthodes : l'approche variationnelle ou l'approche " Programmation Dynamique ».

\subsection{Trajectoires maximales au sens de Pareto}

Supposons à présent que nos joueurs adoptent une attitude plus coopérative c'est-à-dire qu'ils acceptent de se concerter au niveau de la conduite de l'action mais qu'ils préfèrent récolter séparément les fruits de leur participation. Dans ces conditions le partage des résultats découle automatiquement de la stratégie adoptée. Pour éviter de prendre parti " a priori "sur le partage des résultats, les joueurs peuvent convenir d'adopter comme critère de décision la règle de Pareto : une stratégie $A$ sera préférée à la stratégie $B$ aux yeux de la coalition $S$ s'il n'existe aucun joueur pour lequel $B$ est préféré à $A$ et s'il en existe au moins un pour lequel $A$ est préféré à $B$. Pour trouver les stratégies maximales aux 
yeux de la coalition, il faut donc résoudre un problème de commande optimale avec un critère d'évaluation vectoriel.

Plus précisément, si nous désignons par $u=\left[u_{1}, \ldots u_{i}, u_{N}\right]$ le vecteur "stratégie " et par $J=\left[J_{1}, \ldots J_{i}, J_{N}\right]$ le vecteur "critère ", la composante $J_{i}$ étant égale à :

$$
\int_{0}^{T} L_{i}(x, u, t)+\Phi_{i}[x(T), T]
$$

le problème consiste à trouver le vecteur $\hat{u}(t)$ et la trajectoire $\hat{x}(t)$ correspondante de telle sorte que :

a) $\hat{u}(t)$ soit une fonction mesurable, bornée, appartenant à l'ensemble $U$ des commandes admissibles.

b) La relation $J(\hat{x}, \hat{u}, t) \leqslant J(x, u, t)$ implique $J(\hat{x}, \hat{u}, t)=J(x, u, t)$.

A cet effet Da Cunha et Polak [7] ont généralisé les résultats de Pontryagin en énonçant le théorème suivant :

Si $f(x, t, u)$ et $L(x, t, u)$ sont continues en $x$ et en $u$ et continûment différentiables en $x$, alors il existe un vecteur $\mu \in E^{N}, \mu>0$ et une fonction vectorielle $\psi(t) \in E^{N}$, tels que parmi toutes les stratégies admissibles $u \in U$, la stratégie $\hat{u}(t)$ réalise à tout instant $t \in(0, T)$ le maximum de l'Hamiltonien

$$
\mathcal{J}(\psi, \mu, x, u, t)=\sum_{i=1}^{N} \mu_{i} L_{i}(x, u, t)+\sum_{k=1}^{n} \psi_{k}(t) \cdot f(x, u, t)
$$

Cet Hamiltonien est construit à partir du double système d'équations différentielles :

$$
\mid \begin{aligned}
\dot{\psi}(t) & =-\nabla_{x}[\mathcal{H}(u, \psi, \hat{x}, \hat{u}, t)] ; \quad \psi(T)=\sum_{i=1}^{N} \mu_{i} \cdot \frac{\partial \Phi_{i}[\hat{x}(T), T]}{x(T)} \\
\stackrel{\circ}{x} & =f(\hat{x}, \hat{u}, t) ; \quad x(0)=x_{0}
\end{aligned}
$$

Ce résultat concernant les conditions nécessaires d'optimalité, suggère qu'il doit être possible de définir l'ensemble des stratégies maximales en résolvant le problème paramétré :

$$
\begin{aligned}
& P(\mu):\left[\underset{u \in U}{\operatorname{ax}}\left[J[\mu]=\sum_{i=1}^{N} \mu_{i} J_{i}\right]\right. \\
& \text { sous les contraintes } \stackrel{\circ}{x}=f(x, t, u) ; \quad x(0)=x_{0} \\
& \text { et pour toutes les valeurs du vecteur } \mu \text { telles que } \\
& \mu_{i} \geqslant 0 \text { et } \sum_{i=1}^{N} \mu_{i}=1
\end{aligned}
$$


En effet, puisque $\psi$ et $\mu$ ne sont pas identiquement nuls, il n'est pas interdit d'effectuer un changement d'échelle de telle sorte que :

$$
\sum_{i=1}^{N} \mu_{i}=1
$$

Ceci signifie que l'on remplace le critère vectoriel par un critère scalaire dans lequel les objectifs des membres de la coalition sont pondérés par le vecteur $\mu$. Mais en balayant l'ensemble des valeurs de $\mu$, parcourt-on pour autant l'ensemble des stratégies maximales?

Rien ne permet de l'affirmer, car les propriétés requises pour que la solution du problème paramétré coïncide exactement avec l'ensemble des stratégies maximales sont difficiles à énoncer et encore plus difficiles à vérifier. On sait toutefois qu'en résolvant le problème paramétré $P(\mu)$ on est assuré d'obtenir, sinon toutes, tout au moins la plupart des stratégies maximales, et c'est là l'essentiel pour les applications pratiques. Il reste ensuite à choisir à l'intérieur de cet ensemble une stratégie qui soit acceptable par chacun des membres de la coalition.

\subsection{Interprétation économique des jeux différentiels}

\subsubsection{L'intérêt des jeux différentiels dans l'analyse des conflits éco- nomiques}

Il est évident qu'un certain nombre de conflits économiques peuvent être schématisés sous forme d'un jeu différentiel. D'une manière général, les joueurs représentent des centres de décisions économiques. Nous admettrons ici qu'il s'agit d'entreprises ou d'unités de production importantes à l'intérieur d'une même organisation productive. Mais ce pourrait être également l'État, des syndicats de travailleurs, des organismes bancaires ou financiers ou encore des catégories de consommateurs. Le niveau des décisions ou des activités de chaque centre est repéré par le vecteur $u_{i}$ : pour une entreprise, ces décisions concernent les achats, ou les ventes, le programme de production ou d'équipement, la politique financière ou la stratégie commerciale, etc... Quant au système, il représente l'ensemble des phénomènes économiques qui intéressent les organes de décision et sur lesquels ces derniers peuvent exercer leurs activités : il s'agit souvent de stocks de ressources qui sont en la possession ou tout au moins à la disposition des centres de décision, mais ce peut être aussi une position sur un marché ou encore le niveau de certains agrégats économiques. Le champ d'action des différents agents économiques est très variable. L'évolution de certains éléments du système reste quelquefois sous le contrôle exclusif d'un des agents économiques : c'est ainsi que chaque entreprise peut, dans une certaine mesure, choisir sa capacité de production. En revanche, d'autres éléments évoluent sous l'action conjuguée des différents centres de décision : il en est ainsi par exemple de l'intensité des besoins sous l'effet de la publicité, du volume et de la qualification de la main d'œuvre, du niveau et de la répartition de l'épargne ou encore du volume de certains é uipements collectifs. Entre le recouvrement 
total des champs d'action et le morcellement complet du système on peut observer toute une variété de situations qui peuvent être décrites en termes de jeu différentiel. En particulier, dès l'instant où le jeu se déroule dans une économie d'échange, le niveau des ressources détenues par un agent économique dépend toujours partiellement des décisions des autres agents.

Il reste que le recouvrement des champs d'action ne suffit pas à expliquer l'interdépendance spatio-temporelle qui caractérise les conflits économiques. Même dans un système complètement morcelé, on pourrait observer des conflits en raison même des structures d'évaluation. La plupart des agents économiques sont en effet beaucoup plus sensibles à leur situation relative vis-à-vis de leurs adversaires ou de leurs alliés (c'est-à-dire à l'ensemble du vecteur "état ") qu'à leur position absolue (c'est-à-dire aux seules composantes qu'ils contrôlent ou qui leur appartiennent.

L'hypothèse classique d'une économie constituée d'une foule de "Robinson Crusoé " produisant ou consommant chacun de leur côté sans se préoccuper de ce que fait le voisin, apparaît de moins en moins soutenable. Les effets induits deviennent en effet des facteurs déterminants dans la conduite de l'action : ils sont à la source du comportement des consommateurs, des luttes de prestige que se livrent certaines entreprises et des conflits sociaux-économiques qui opposent les plus démunis aux plus favorisés.

Il est très rare que le champ d'appréciation d'un centre de décision coïncide a vec son champ d'action et qu'il n'y ait pas de recouvrement des champs d'appréciation des différents acteurs économiques. C'est ce double phénomène de recouvrement qui explique toute l'importance que revêt la théorie des jeux différentiels pour analyser la dynamique des conflits économiques.

\subsubsection{Interprétation économique des techniques de séparation et des fonctions duales associées}

La technique d'analyse est directement inspirée de la théorie de la commande optimale. Il s'agit, dans toute la mesure du possible, de remplacer le conflit dynamique par une suite de conflits statiques. Dans ce but, chaque joueur s'efforce de dresser à tout instant un bilan aussi complet que possible de son action en tenant compte des transformations qu'il provoque, transformations qui peuvent améliorer ou au contraire détériorer sa position relative, accroître ou diminuer ses potentialités.

Ici, comme dans la théorie de la commande optimale, les transformations éventuelles $\stackrel{\circ}{x}$ sont évaluées sur la base d'un système de valorisation marginale $\psi_{i}$. Mais dans un schéma conflictuel, il existe autant de systèmes de valorisations $\psi_{i}$ qu'il existe de joueurs ou de coalitions en présence puisque chacun apprécie l'évolution du système au travers des objectifs personnels qu'il poursuit. Chaque composante $\mathrm{du}$ vecteur $\psi_{i}(t)$ représente donc l'utilité marginale future que le joueur (i) attribue au fait de disposer à 
l'instant $t$ d'une situation un peu plus favorable sur la composante $x(t)$ correspondante, toutes choses restant égales par ailleurs. En particulier si $x(t)$ représente des stocks de ressources le fait de disposer à l'instant $t$ d'un stock supplémentaire de marchandise $(p)$ permettra à l'agent (i) d'augmenter ses performances dans le futur (par exemple augmenter son bénéfice ou diminuer ses charges). La composante $(p)$ du vecteur $\psi_{i}(t)$ mesure cette augmentation et révèle du même coup l'usage que l'agent $(i)$ pourrait faire de cette ressource supplémentaire. Le vecteur $\psi_{i}(t)$ peut ainsi être interprété comme le prix d'usage du système pour le joueur (i). La définition d'un Hamiltonien pour chaque joueur ou pour chaque coalition vise donc à exprimer le rapport de force qui existe à une époque donnée entre les différentes parties en présence lorsque celles-ci sont conscientes de leurs intérêts à long terme. Toute la difficulté consiste précisément à estimer les différents vecteurs de valorisations marginales. En effet, si les positions ou les potentialités respectives sont affaire d'appréciation personnelle, l'usage que chacun peut en faire dépend des stratégies des autres joueurs. En d'autres termes, l'utilité marginale future $\psi_{i}$ des ressources détenues ou contrôlées à un instant donné par le joueur (i) dépend bien entendu des objectifs qu'il poursuit mais aussi de la manière dont le jeu va se dérouler. C'est pourquoi les équations qui régissent le système de prix d'usage $\psi_{i}(i=1 \ldots N)$ dépendent non seulement de l'attitude adoptée (attitude de défense, attitude de compromis, attitude coopérative) mais aussi de l'information disponible (hypothèse de régulation en boucle ouverte ou de régulation en boucle fermée).

Dans le cas d'un duel, les deux joueurs apprécient évidemment de manière symétrique l'évolution du système, ce qui se traduit par des prix d'usage égaux mais de signes contraires.

Dans l'optique coopérative, le vecteur prix d'usage est commun à tous les membres d'une même coalition mais il dépend du poids respectif de chacun des joueurs dans la conduite de l'action.

Enfin, dans l'optique non coopérative, le long de la trajectoire d'équilibre, il existe pour chaque groupe d'intérêt un vecteur de valorisation marginale différent qui témoigne de la diversité des objectifs et d'une certaine appréciation de l'évolution du conflit. Tout se passe comme si chaque agent économique était assisté d'un "ange gardien " capable de lui révéler les conséquences futures de ses initiatives. Il va de soi que tous les éléments du système qui se situent hors du champ d'appréciation d'un agent ont pour cet agent un prix d'usage nul, même si ces éléments appartiennent à son domaine d'action. Par ailleurs on admettra volontiers que si chaque agent est informé à tout instant de sa situation et de celle de ses rivaux (hypothèse de régulation en boucle fermée) son système de. valorisation doit tenir compte des réactions éventuelles de ces derniers. Ceci étant, l'interprétation économique des jeux différentiels apparaît comme un simple prolongement de l'interprétation économique du Principe du Maximum. 


\section{LE JEU DIFFERENTIEL LINEAIRE-QUADRATIQUE}

Nous venons de voir que le calcul des solutions d'équilibre ou des solutions maximales est extrêmement lourd puisqu'il impose, soit de trouver le point d'équilibre d'un certain nombre de duels (équilibre minimax), soit de résoudre un problème de commande optimale paramétré (solution d'équilibre de Nash-Cournot), ce qui devient inextricable.

Dans ce dernier cas, on peut imaginer d'alléger les calculs en s'appuyant sur les conditions caractéristiques énoncées au paragraphe précédent. Mais il ne faut pas se dissimuler que dans l'état actuel des connaissances, l'approche variationnelle comme l'approche " programmation dynamique " sont toutes deux impuissantes à nous fournir la solution d'équilibre du système par une procédure itérative. Et cela tient tout simplement au fait qu'à l'équilibre les termes $\frac{\partial \mathcal{H}_{i}}{\partial u_{j}}$ ne sont pas nuls.

Le calcul se trouve cependant facilité lorsqu'on étudie certains schémas particuliers (duel, organisation tendue vers un même but, organisation décomposable, organisation hiérarchisée) ou encore lorsque le système revêt une forme spécifique (évolution linéaire et fonctions économiques quadratiques).

A titre d'application de la théorie exposée au paragraphe précédent, nous analyserons donc cette forme particulière. A cet effet, considérons le jeu suivant appelé " jeu linéaire-quadratique » :

$$
\mid \begin{aligned}
& \underset{u_{i}}{\operatorname{Max}}\left[J_{i}\right] \quad i=1 \ldots N \\
& \operatorname{avec} \stackrel{\circ}{x}=A x+\sum_{j=1}^{N} B_{j} u_{j} ; x(0)=x_{0} \\
& J_{i}=\frac{1}{2} \int_{0}^{T}\left[x^{\prime} P_{i} x+\sum_{j=1}^{N} u_{j}^{\prime} Q_{i j} u_{j}\right] \mathrm{d} t+\frac{1}{2} x^{t}(T) R_{i} x(T)
\end{aligned}
$$

$A, B, P_{i}, Q_{i}, R_{i}$ étant des fonctions du temps connues de l'ensemble des joueurs et l'indice supérieur "prime " désignant les matrices ou vecteurs transposés.

\subsection{Trajectoires minimax}

Nous savons déjà qu'on obtient la stratégie de défense ou stratégie minimax de chaque joueur en résolvant un jeu à somme nulle.

\subsubsection{Approche variationnelle}

Pour ce faire, considérons l'Hamiltonien du joueur $(i)$.

$$
\mathfrak{H e}_{i}=\frac{1}{2}\left[x^{\prime} P_{i} x+\sum_{j=1}^{N} u_{j}^{\prime} Q_{i j} u_{j}\right]+\Psi_{i}^{\prime \prime}\left[A x+\sum_{j=1}^{N} B_{j} u_{j}\right]
$$


On obtient immédiatement les conditions d'équilibre du duel entre le joueur $(i)$ et la coalition des autres joueurs :

a)

$$
\begin{aligned}
& \stackrel{\circ}{\Psi}_{i}=-P_{i} x-A^{\prime} \bar{\psi}_{i} ; \quad \bar{\psi}_{i}(T)=R_{i} x(T) \\
& Q_{i i} \bar{u}_{i}+B_{i}^{\prime} \bar{\psi}_{i}=0 \\
& Q_{i j} \tilde{u}_{j}+B_{j}^{\prime} \bar{\psi}_{i}=0 \quad \text { pour } j \neq i
\end{aligned}
$$

b) $Q_{i i}$ définie négative (recherche d'un maximum)

$Q_{i j}$ définie positive $\forall j \neq i$ (recherche d'un minimum) ce qui permet d'écrire la stratégie minimax du joueur $(i)$

$$
\bar{u}_{i}=-Q_{i i}^{-1} B_{i}^{\prime} \bar{\psi}_{i}
$$

et la stratégie présumée de la coalition :

$$
\hat{u}_{j}=-Q_{i j}^{-1} B_{j}^{\prime} \bar{\psi}_{i} ; \quad \forall j \neq i
$$

Le prix d'usage $\bar{\psi}_{i}$ relatif à la stratégie minimax obéit au double système d'équation :

$$
\left[\begin{array}{ll}
\stackrel{\circ}{\Psi}_{i}=-P_{i} x-A^{\prime} \bar{\psi}_{i}, & \bar{\psi}_{i}(T)=R_{i} x(T) \\
\stackrel{\circ}{x}=A x-\sum_{j=1}^{N} B_{j} Q_{i j}^{-1} B_{j}^{\prime} \bar{\psi}_{i} ; & x(0)=x_{0}
\end{array}\right.
$$

Pour résoudre ce double système d'équation, on étudie une solution de la forme :

$$
\bar{\psi}_{i}(t)=\bar{\alpha}_{i}(t) x(t)
$$

dans laquelle $\bar{\alpha}_{i}$ est une matrice carrée de même dimension que le vecteur " état".

On montre alors facilement que les conditions précédentes sont satisfaites si $\alpha_{i}(t)$ est solution du système

$$
\mid \begin{aligned}
& \stackrel{\circ}{\alpha}_{i}=-\bar{\alpha}_{i} A-A^{\prime} \bar{\alpha}_{i}-P_{i}+\bar{\alpha}_{i} \sum_{j=1}^{N} B_{j} Q_{i j}^{-1} B_{j}^{\prime} \bar{\alpha}_{i} \\
& \bar{\alpha}_{i}(T)=R_{i}
\end{aligned}
$$

Cet ensemble d'équations différentielles quadratiques permet de calculer la matrice symétrique $\bar{\alpha}_{i}$ et partant de là, la stratégie minimax

$$
\bar{u}_{i}=-Q_{i i}^{-1} B_{i}^{\prime} \bar{\alpha}_{i} \cdot x
$$

En adopiant cette stratégie, le résultat minimum que peut obtenir le joueur (i) cst égal à

$$
J_{i}\left(x_{0}, 0\right)=\frac{1}{2} x_{0}^{\prime} \bar{\alpha}_{i}(0) x_{0}
$$


En effet, on peut démontrer que :

$$
\begin{aligned}
J_{i}[x(t), t] & =\frac{1}{2} \int_{t}^{T}\left[x^{\prime} P_{i} x+\bar{u}_{i} Q_{i i} \bar{u}_{i}+\sum_{j \neq i} \tilde{u}_{j} Q_{i j} \tilde{u}_{j}\right] \mathrm{d} t \\
& +\frac{1}{2} x^{\prime}(t) R_{i} x(T)
\end{aligned}
$$

est égal à la forme quadratique :

$$
\overline{\frac{1}{2} x^{\prime} \bar{\alpha}_{i}(t) x}
$$

Il suffit pour le vérifier de différencier l'équation précédente par rapport au temps : on retrouve alors les expressions de $\frac{\circ}{\alpha_{i}}$ et de $\bar{\alpha}_{i}(T)$.

\subsubsection{Approche « Programmation dynamique »}

On part de l'équation d'Hamilton-Jacobi-Bellman :

$$
\begin{aligned}
-\frac{\partial J_{i}}{\partial t} & =\operatorname{Max}_{u_{i}}\left[\begin{array}{c}
\frac{1}{2} x^{\prime} P_{i} x+\frac{1}{2} u_{i}^{\prime} Q_{i i} u_{i}+\frac{1}{2} \sum_{j \neq i} u_{j}^{\prime} Q_{i j} u_{j} \\
+\frac{1}{2}\left[\frac{\partial J_{i}}{\partial x}\right] \stackrel{o}{x}+\frac{1}{2} \stackrel{o}{\prime}^{\prime}\left[\frac{\partial J_{i}}{\partial x}\right]^{\prime}
\end{array}\right] \\
& =\operatorname{Min}_{u j}[\text { la même expression] }
\end{aligned}
$$

avec $: J_{i}[x(T), T]=\frac{1}{2} x^{\prime}(T) R_{i} x(T)$.

Les stratégies d'équilibre sont alors :

$$
\bar{u}_{i}=-Q_{i i}^{-1} B_{i}^{\prime}\left[\frac{\partial J_{i}}{\partial x}\right]^{\prime} ; \quad \tilde{u}_{j}=-Q_{i j}^{-1} B_{j}^{\prime}\left[\frac{\partial J_{i}}{\partial x}\right]^{\prime}
$$

Pour résoudre cette équation, le caractère quadratique du problème nous suggère d'étudier une solution de. la forme

$$
\bar{J}_{i}(x, t)=\frac{1}{2} x^{\prime} \vec{\beta}_{i} x
$$

On vérifie alors immédiatement que l'équation d'Hamilton-JacobiBellman est satisfaite à condition que $\beta_{i}(t)$ soit solution du système

$$
\mid \begin{aligned}
& \stackrel{\circ}{\beta}_{i}=-A^{\prime} \bar{\beta}_{i}-\bar{\beta}_{i} A+\bar{\beta}_{i} \sum_{j=1}^{N} B_{j} Q_{i j}^{-1} B_{j}^{\prime} \bar{\beta}_{i} \\
& \bar{\beta}_{i}(T)=R_{i}
\end{aligned}
$$


Puisque la matrice $\bar{\beta}_{i}(t)$ satisfait au même système d'équations différentielles que la matrice $\bar{\alpha}_{i}(t)$ et que leurs valeurs à l'horizon $T$ sont identiques, $\bar{\beta}_{i}(t)=\bar{\alpha}_{i}(t)$.

Par ailleurs, puisque $\frac{\partial \bar{J}_{i}}{\partial x}=x^{\prime} \bar{\beta}_{i}$ et $\bar{\psi}_{i}(t)=\bar{\alpha}_{i} x$

$$
\bar{\psi}_{i}^{\prime}(t)=\frac{\partial \bar{J}_{i}}{\partial x}
$$

ce qui fonde l'interprétation économique que nous avons déjà donnée.

\subsection{Trajectoire d'équilibre de Nash-Cournot}

\subsubsection{Régulation en boucle ouverte}

Dans ce type de régulation, le joueur $(i)$ considère que la stratégie $u_{j}$ des autres joueurs est une fonction du temps. Ici encore le problème peut être résolu soit par l'approche variationnelle, soit par la " Programmation Dynamique "; mais dans le cas d'une régulation en boucle ouverte, la première reste de loin la plus simple.

On écrit en effet l'Hamiltonien du joueur (i) $\mathcal{H}_{i}=\frac{1}{2} x^{\prime} P_{i} x+\frac{1}{2} u_{i}^{\prime} Q_{i i} u_{i}+\frac{1}{2} \sum_{j \neq i} u_{j}^{* \prime} Q_{i j} u_{j}^{*}$

$$
+\psi_{i}^{\prime}\left[A x+B_{i} u_{i}+\sum_{j \neq i} B_{j} u_{j}^{*}\right]
$$

On obtient alors immédiatement les conditions :

$$
\mid \begin{array}{ll}
\text { (a) } \quad \dot{\Upsilon}_{i}^{*}=-P_{i} x-A^{\prime} \psi_{i}^{*} \\
& Q_{i i} u_{i}^{*}+B_{i}^{\prime} \psi_{i}^{*}=0 \\
\text { (b) } & Q_{i i} \text { définie négative }
\end{array}
$$

ce qui détermine la stratégie d'équilibre du joueur $(i)$ :

$$
u_{i}^{*}=-Q_{i i}^{-1} B_{i}^{\prime} \psi_{i}^{*}
$$

Par ailleurs, si tous les centres de décision jouent leur stratégie d'équilibre, l'équation d'évolution devient :

$$
\stackrel{\circ}{x}=A x-\sum_{j=1}^{N} B_{j} Q_{j j}^{-1} B_{j}^{\prime} \psi_{j}^{*}
$$

Pour résoudre le double système d'équations différentielles $\left(\stackrel{\circ}{x}, \stackrel{\vartheta}{\psi}_{1} \forall_{i}\right)$ on peut comme au paragraphe précédent essayer une solution de la forme :

$$
\psi_{i}^{*}(t)=\alpha_{i}^{*}(t) x(t)
$$

dans laquelle $\alpha_{i}^{*}$ est une matrice carrée de même dimension que le vecteur " état". 
On démontre alors que les équations différentielles $\left(\stackrel{\circ}{x}, \stackrel{\bigotimes}{i}_{i}\right)$ sont satisfaites lorsque la matrice $\alpha_{i}^{*}(t)$ est solution du système :

$$
\mid \begin{aligned}
& \dot{\alpha}_{i}^{*}=-A^{\prime} \alpha_{i}^{*}-\alpha_{i}^{*} A-P_{i}+\alpha_{i}^{*} \sum_{j=1}^{N} B_{j} Q_{j j} B_{j}^{\prime} \alpha_{j}^{*} \\
& \alpha_{i}^{*}(T)=R_{i}
\end{aligned}
$$

On notera immédiatement qu'il s'agit, comme au paragraphe précédent, d'équations différentielles quadratiques mais que nous sommes maintenant en présence d'un véritable système couplé (interdépendance entre ${ }^{*}{ }_{i}$ et ${ }_{\alpha}^{*}$ ).

Le long de la trajectoire d'équilibre, le résultat du joueur $(i)$ peut être obtenu en essayant une solution de la forme

$$
J_{i}^{*}[x(t), t]=\frac{1}{2} x^{\prime} \beta_{i}^{*} x
$$

En différenciant l'égalité

$$
\frac{1}{2} x^{\prime} \beta_{i}^{*} x=\frac{1}{2} \int_{t}^{T}\left[x^{\prime} P_{i} x+\sum_{j=1}^{N} u_{j}^{* \prime} Q_{i j} u_{j}^{*}\right] \mathrm{d} t+\frac{1}{2} x^{t}(T) R_{i} x(T)
$$

on obtient :

$$
\mid \begin{aligned}
& \stackrel{\circ}{\beta}_{i}^{*}=-A^{\prime} \beta_{i}^{*}-\beta_{i} A+\sum_{j=1}^{N} \beta_{i} B_{j} Q_{j j}^{-1} B_{j}^{\prime} \alpha_{j} \\
&+\sum_{j=1}^{N} \alpha_{j}^{\prime} B_{j} Q_{j j}^{-1} B_{j}^{\prime} \beta_{i}+\sum_{j=1}^{N} \alpha^{\prime} B_{j} Q_{j j}^{-1} Q_{i j}^{-1} Q_{j j}^{-1} B_{j}^{\prime} \alpha_{j} \\
& \beta_{i}^{*}(T)=R_{i}
\end{aligned}
$$

Ce nouveau système d'équations différentielles quadratiques non couplées fournit généralement une solution $\beta_{i}^{*}(t)$ différente de $\alpha_{i}^{*}(t)$. Cette différence tient essentiellement au caractère disymétrique de la matrice $\alpha_{i}^{*}(t)$.

L'approche programmation dynamique permet d'ailleurs de se rendre compte de manière plus explicite de cette différence. Considérons à cet effet l'équation d'Hamilton-Jacobi-Bellman :

$$
\left.\begin{array}{c}
-\frac{\partial J_{i}^{*} x(t), t}{\partial t}=\operatorname{Max}_{u_{i}}\left[\begin{array}{c}
\frac{1}{2} x^{\prime} P_{i} x+\frac{1}{2} u_{i} Q_{i i} u_{i}+\frac{1}{2} \sum_{j \neq i} u_{j}^{*}(t) Q_{i j} u_{j}^{*}(t) \\
+\frac{1}{2}\left[\frac{\partial J}{\partial x}\right] \stackrel{\circ}{x}+\frac{1}{2} \stackrel{\circ}{x}^{\prime}\left[\frac{\partial J}{\partial x}\right]^{\prime}
\end{array}\right] \\
J_{i}[x(T), T]=\frac{1}{2} x^{\prime}(T) R_{i} x(T)
\end{array}\right]
$$


et une solution de la forme :

$$
J_{i}^{*}[x(t), t]=\frac{1}{2} x^{\prime} \quad \Upsilon_{i}(t) \quad x+\delta_{i}^{\prime}(t) x+\varepsilon_{i}(t)
$$

dans laquelle $\gamma_{i}$ est une matrice symétrique.

On montre alors que l'équation d'Hamilton-Jacobi est vérifiée si :

$$
u_{i}^{*}(t)=-Q_{i i}^{-1} B_{i}^{\prime}\left[\gamma_{i} x+\delta_{i}\right]
$$

avec :

$$
\begin{aligned}
& {\stackrel{\circ}{\gamma_{i}}}^{\prime}=-\gamma_{i} A-A^{\prime} \gamma_{i}-P_{i}+\gamma_{i} B_{i} Q_{i i}^{-1} B_{i}^{\prime} \gamma_{i} ; \quad \gamma_{i}(T)=R_{i} \\
& \stackrel{\circ}{\delta}_{i}=-A^{\prime} \delta_{i}+\gamma_{i} B_{i} Q_{i i}^{-1} B_{i}^{\prime} \delta_{i}-\gamma_{i} \sum_{j \neq i} B_{j} u_{j}^{*} ; \delta_{i}(T)=0 \\
& {\stackrel{\circ}{\varepsilon_{i}}}^{\prime}=\frac{1}{2} \delta_{i}^{\prime} B_{i} Q_{i i}^{-1} B_{i}^{\prime} \delta_{i}-\sum_{j \neq i} \delta^{\prime} B_{j} u_{j}^{* \prime}-\frac{1}{2} \sum_{j \neq i} u_{j}^{* \prime} Q_{i j} u_{j} ; \quad \varepsilon_{i}(T)=0
\end{aligned}
$$

Si tous les joueurs adoptent leur stratégie d'équilibre, l'équation d'évolution s'écrit :

$$
\stackrel{\circ}{x}=A x-\sum_{j=1}^{N} B_{j} R_{j j}^{-1} B_{j}^{\prime}\left[\gamma_{j} x+\delta_{j}\right]
$$

et on constate que l'équation différentielle $\left(\AA_{i}\right)$ dépend linéairement de $x$.

Ceci nous suggère de poser $\delta_{i}(t)=\underline{p_{i}(t) x ; p_{i}(t) \text { étant une matrice }}$ carrée de même dimension que le vecteur "état".

On aboutit alors au système :

$$
\mid \begin{aligned}
& \stackrel{\circ}{\rho}_{i}=-\rho_{i} A-A^{\prime} \rho_{i}+\left(\rho_{i}+\gamma_{i}\right) \sum_{j=1}^{N} B_{j} Q_{j j}^{-1} B_{j}^{-1}\left(\rho_{i}+\gamma_{i}\right)-\gamma_{i} B_{i} Q_{i i}^{-1} B_{i}^{\prime} \gamma_{i} \\
& \rho_{i}(T)=0
\end{aligned}
$$

Il suffit alors d'ajouter les expressions de $\dot{\gamma}_{i}$ et $\dot{\rho}_{i}$ pour constater que la matrice $\alpha_{i}^{*}(t)$ est obtenue par addition de la matrice $\gamma_{i}(t)$ et de la matrice dissymétrique $\rho_{i}(t)$.

Ce calcul élémentaire montre par ailleurs que :

$$
\frac{\partial J_{i}^{*}}{\partial x}=x^{\prime} \gamma_{i}(t)+\delta_{i}^{\prime}(t)=x^{\prime}\left[\gamma_{i}+\rho_{i}^{\prime}\right]=x^{\prime} \alpha_{i}^{* \prime}=\psi_{i}^{* \prime}
$$

On retrouve ainsi l'interprétation variationnelle des fonctions duales $\psi_{i}^{*}$.

En écrivant l'expression de $\stackrel{\odot}{i}_{i}$ en fonction de $\rho_{i}$ et $\gamma_{i}$ on montrerait facilement que $\varepsilon_{i}(t)$ est une forme quadratique du type :

$$
\varepsilon_{i}(t)=\frac{1}{2} x^{\prime} \tau_{i} x
$$


dans laquelle la matrice $\tau_{i}$ est une matrice symétrique. De ce fait, comme

$$
J_{i}^{*}[x(t), t]=\frac{1}{2} x^{\prime}\left[\gamma_{i}+2 \rho_{i}^{\prime}+\tau_{i}\right] x=\frac{1}{2} x^{\prime}\left[\gamma_{i}+\rho_{i}+\rho_{i}^{\prime}+\tau_{i}\right] x
$$

il suffit de poser

$$
\beta_{i}^{*}=\gamma_{i}+\rho_{i}^{\prime}+\rho_{i}+\tau_{i}
$$

pour retrouver le résultat :

$$
J_{i}^{*}[x(t), t]=\frac{1}{2} x^{\prime} \beta_{i}^{*}(t) \cdot x
$$

Dans un jeu à deux joueurs à somme nulle

$$
P=P_{1}=P_{2} ; \quad Q_{12}=-Q_{22} ; \quad Q_{21}=-Q_{11} ; \quad R_{1}=-R_{2}=R
$$

On démontre alors très facilement qu'il existe une valeur commune

$$
\alpha^{*}=\alpha_{1}^{*}=\beta_{1}^{*}=-\alpha_{2}^{*}=-\beta_{2}^{*}
$$

et que le gain du 1 er joueur (égal à la perte du $2^{\mathrm{e}}$ joueur) peut s'écrire :

$$
J_{i}^{*}=-J_{2}^{*}=\frac{1}{2} x^{\prime} \alpha^{*}(t) x
$$

Dans ce cas la matrice symétrique $\alpha^{*}(t)$ est solution de l'équation :

$$
\begin{aligned}
& \AA^{*}=-A^{\prime} \alpha^{*}-\alpha^{*} A-P+\alpha^{*}\left[B_{1} Q_{11}^{-1} B_{1}^{\prime}-B_{2} Q_{22}^{-1} B_{2}^{\prime}\right] \alpha^{*} \\
& \alpha^{*}(T)=R
\end{aligned}
$$

\subsubsection{Régulation en boucle fermée}

En utilisant l'approche variationnelle $\left({ }^{1}\right)$, les conditions d'équilibre s'écrivent à présent :

a)

$$
\begin{aligned}
& \dot{\vartheta}_{i}^{*}=-P_{i} x-A^{\prime} \psi_{i}^{*}-\sum_{j=1}^{N}\left[\frac{\partial u_{j}^{*}}{\partial x}\right]^{\prime} Q_{i j} u_{j}^{*}-\sum_{j=1}^{N}\left[\frac{\partial u_{j}^{*}}{\partial x}\right]^{\prime} B_{j}^{\prime} \psi_{i} \\
& Q_{j j} u_{j}^{*}+B_{j}^{\prime} \psi_{j}^{*}=0 \quad, \quad \forall j=(1 \ldots N)
\end{aligned}
$$

b) $Q_{j j}$ définie négative, $\forall j=(1 \ldots N)$

$$
\stackrel{\circ}{x}=A x-\sum_{j=1}^{N} B_{j} Q_{i j}^{-1} B_{j} \psi_{j}^{*}
$$

En boucle ouverte comme en boucle fermée, l'évolution du système est donc régie par une équation de même forme. Seule change l'équation différentielle qui gouverne l'évolution des prix d'usage $\psi_{i}$.

(1) Nous laissons le soin au lecteur de retrouver les résultats de ce paragraphe à partir de l'équation d'Hamilton-Jacobi. 
Cette remarque nous incite à poser comme précédemment $\psi_{i}^{*}(t)=\beta_{i}^{*}(t) x$, la matrice $\beta_{i}^{*}$ ayant bien entendu une détermination différente.

On obtient alors :

$$
\begin{aligned}
u_{j}^{*}= & -Q_{j j}^{-1} B_{j}^{\prime} \beta_{j}^{*} x \\
\stackrel{\psi}{i}_{i}^{*}= & -P_{i} x-A^{\prime} \psi_{i}^{*}-\sum_{j=1}^{N} \beta_{j}^{* *} B_{j} Q_{j j}^{-1} B_{j}^{\prime} \psi_{j}^{*} \\
& -\sum_{j=1}^{N} \beta_{j}^{\prime *} B_{j} Q_{j j}^{-1} Q_{i j} Q_{j j}^{-1} B_{j}^{\prime} \psi_{j}^{*}
\end{aligned}
$$

et la matrice $\beta_{i}^{*}$ apparaît comme la solution d'un système couplé d'équations différentielles quadratiques :

$$
\mid \begin{aligned}
& \stackrel{\circ}{\beta}_{i}^{*}=-A^{\prime} \beta_{i}^{*}-\beta_{i}^{*} A-P_{i}+\sum_{j=1}^{N} \beta_{i}^{* \prime} B_{j} Q_{j j}^{-1} B_{j}^{\prime} \beta_{j}^{*} \\
&+\sum_{j=1}^{N} \beta_{j}^{* \prime} B_{j} Q_{j j}^{-1} B_{j}^{\prime} \beta_{i}^{*}-\sum_{j=1}^{N} \beta_{j}^{* \prime} B_{j} Q_{j j}^{-1} Q_{i j} Q_{j j}^{-1} B_{j}^{\prime} \beta_{j}^{*} \\
& \beta_{i}(T)=R_{i}
\end{aligned}
$$

Le lecteur pourra vérifier facilement que la matrice $\beta_{i}^{*}$ est ici une matrice symétrique $\left(\beta_{i}^{*}=\beta_{i}^{\prime *}\right)$ et que le résultat du joueur $(i)$ s'écrit très simplement

$$
J_{i}^{*}[x(t), t]=\frac{1}{2} x^{\prime} \beta_{i}^{*}(t) x
$$

On en déduit immédiatement que :

$$
\psi_{i}^{* \prime}(t)=x^{\prime} \beta_{i}^{* \prime}=x^{\prime} \beta_{i}^{*}=\frac{\partial J^{*}}{\partial x}
$$

Pour ce qui concerne les prix d'usage $\downarrow_{i}$, la matrice $\beta_{i}^{*}$ en boucle fermée joue donc le même rôle que la matrice $\alpha_{i}^{*}$ en boucle ouverte.

Pour ce qui concerne le résultat $J_{i}^{*}$, elle joue le même rôle que la matrice $\beta_{i}^{*}$ en boucle ouverte.

Mais les équations différentielles qui régissent l'évolution de ces trois matrices ne sont pas les mêmes, ce qui explique les écarts importants que l'on peut oberver entre les trajectoires d'équilibre en boucle ouverte et en boucle fermée.

Ces trois matrices ne coïncident en effet que dans deux cas particuliers :

$1^{\circ}$ Lorsqu'il existe un centre de commande unique. On sait alors que la matrice $\alpha^{*}=\beta^{*}$ obéit à l'équation de Riccati :

$$
\dot{\alpha}^{*}=-A^{\prime} \alpha^{*}-\alpha^{*} A-P+\alpha^{*} B Q^{-1} B^{\prime} \alpha^{*} ; \quad \alpha^{*}(T)=R
$$


$2^{\circ}$ Dans un jeu à deux joueurs à somme nulle. On sait alors que la matrice $\alpha^{*}=\alpha_{1}^{*}=\beta_{1}^{*}=-\alpha_{2}^{*}=-\beta_{2}^{*}$ est une solution de l'équation de Riccati :

$$
\begin{aligned}
& \stackrel{0}{\alpha}^{*}=-A^{\prime} \alpha^{*}-\alpha^{*} A-P+\alpha^{*}\left[B_{1} Q_{11}^{-1} B_{1}^{\prime}=B_{2} Q_{22}^{-1} B_{2}^{\prime}\right] \alpha^{*} \\
& \alpha^{*}(T)=R
\end{aligned}
$$

avec $: P=P_{1}-P_{2}, Q_{22}=-Q_{12} ; Q_{11}=-Q_{21} ; R=R_{1}=-R_{2}$.

\subsection{Trajectoires maximales au sens de Pareto}

Nous savons déjà qu'il est extrêmement difficile de calculer toutes les solutions maximales au sens de Pareto mais qu'il est possible d'obtenir un certain nombre d'entre elles en résolvant le problème de commande optimale paramétré

$$
\begin{gathered}
\underset{\substack{\operatorname{Max} \\
u_{1} u_{2}, u_{N}}}{\stackrel{\circ}{x}=A x+\sum_{i=1}^{N} B_{i} u_{i}} \\
\operatorname{avec} \mu_{i} \geqslant 0 \text { et } \sum_{i=1}^{N} \mu_{i}=1
\end{gathered}
$$

Les conditions d'optimalité s'écrivent immédiatement :

a)

$$
\begin{aligned}
& \hat{u}_{i}=-\left[\sum_{j=1} \mu_{j} Q_{j i}\right]^{-1} B_{i}^{\prime} \hat{\psi}(\mu) \\
& \dot{\hat{\psi}}=-\sum_{i=1}^{N} \mu_{i} P_{i} x-A^{\prime} \cdot \hat{\psi}(\mu)
\end{aligned}
$$

b)

$$
\sum_{j=1}^{N} \mu_{j} Q_{j i} \text { définie négative }
$$

En posant $\hat{\psi}(\mu)=\hat{\alpha}(\mu) x$, on montre que les conditions ci-dessus sont satisfaites lorsque $\hat{\alpha}(t)$ obéit à l'équation de Riccati :

$$
\begin{aligned}
& \stackrel{\dot{\alpha}}{\alpha}=-\hat{\alpha} A-A^{\prime} \hat{\alpha}-\sum_{i=1}^{N} \mu_{i} P_{i}+\tilde{\alpha} \sum_{i=1}^{N} B_{i} \hat{\rho}_{i} \\
& \hat{\alpha}(T)=\sum_{i=1}^{N} \mu_{i} R_{i} \text { avec } \hat{p}_{i}=\left[\sum_{j=1}^{N} \mu_{j} Q_{j-i}\right]^{-1} B_{i}^{\prime} \hat{\alpha}
\end{aligned}
$$

Par ailleurs, à l'époque $t$, le résultat global que la coalition peut espérer atteindre dans le futur est égal à :

$$
\tilde{J}\left[x(t), t, \mu^{\prime}\right]=\frac{1}{2} x^{\prime}(t) \hat{\alpha} x(t)
$$


Nous laissons au lecteur le soin de calculer la part $J_{i}$ qui reviendra automatiquement au joueur (i) s'il utilise la stratégie maximale $\hat{u}_{i}=-\hat{p}_{i} x$.

\subsection{Extensions}

Les résultats du jeu différentiel linéaire-quadratique que nous venons de présenter ont l'avantage d'être opératoires. Ils mériteraient cependant d'être étendus et on peut imaginer trois types de perfectionnement.

Le premier consiste à étudier une fonction d'évaluation de type quadratique dans laquelle on trouverait, à côté des termes $\frac{1}{2} x^{\prime} P_{i} x$ et $\frac{1}{2} u_{j}^{\prime} Q_{i j} u_{j}$, des termes linéaires et des termes quadratiques croisés entre les vecteurs $\left(x, u_{1}, u_{i}, u_{N}\right)$. Ce perfectionnement alourdirait sensiblement les expressions précédentes mais ne modifierait pas fondamentalement les conclusions que nous venons d'énoncer.

Le second se propose d'étendre les résultats du jeu linéaire quadratique à une classe de jeux assez voisine du type

$$
\begin{aligned}
J_{i}= & \int_{0}^{T}\left[\frac{1}{2} x^{\prime} P_{i} x+\frac{1}{2} \sum_{j=1}^{N} u_{j}^{\prime} Q_{i j} u_{j}+\varepsilon L_{i}\left(x, t, u_{1} \ldots u_{i,} u_{n}\right) \mathrm{d} t\right. \\
& +\frac{1}{2} x^{\prime}(T) R_{i} x(T)+\varepsilon \Phi_{i}[x(T), T] \\
\stackrel{\circ}{x}= & A x+\sum_{j=1}^{N} B_{j} u_{j}+\varepsilon f\left(x, u_{1} \ldots u_{N}\right) ; \quad x(0)=x_{0}
\end{aligned}
$$

dans lequel $\varepsilon$ est un scalaire relativement petit.

Par des développements en série de Taylor en $\varepsilon$, A. W. Starr [9] est parvenu à calculer les corrections qu'il convenait d'apporter à la trajectoire nominale pour tenir compte des écarts $\varepsilon L_{i}$ et $\varepsilon f$.

Toutefois, l'extension la plus intéressante consiste à introduire dans le modèle un certain nombre de contraintes, qu'il s'agisse de contraintes portant exclusivement sur les commandes ou de contraintes mixtes étatcommande. Les premières alourdissent les expressions mais se prêtent assez bien au calcul. Les secondes, en revanche, sont beaucoup plus difficiles à manier car les variables duales correspondantes interviennent directement dans l'équation d'évolution des prix d'usage $\psi_{i}$. Divers algorithmes ont été proposés, mais les expériences ne sont pas encore assez nombreuses pour qu'on puisse en tirer des enseignements concluants.

\section{EXEMPLES ET ORGANISATIONS PARTICULIERES}

\subsection{Quelques organisations particulières}

Indépendamment $\mathrm{du}$ jeu linéaire-quadratique que nous venons d'analyser, les seuls schémas qui se prêtent à l'heure actuelle au calcul concernent toujours des organisations particulières. 
- Il s'agit tout d'abord du jeu à deux personnes à somme nulle à propos duquel on connaît les conditions d'existence et d'unicité de la trajectoire d'équilibre [2] et à propos duquel on dispose d'un certain nombre de méthodes de résolution

- Il en va de même de l'organisation décomposable :

$$
\begin{gathered}
\underset{\left[u_{1}, u_{i}, u_{N}\right]}{\operatorname{Max}}\left[\int_{0}^{T} \sum_{i=1}^{N} L^{i}\left(x_{i}, u_{i}, t\right) \mathrm{d} t+\sum_{i=1}^{N} \Phi_{i}[x(T), T]\right] \\
{\left[\begin{array}{l}
\ddot{x}_{i}=f_{i}\left(x_{i}, t, u_{i}\right) \quad x_{i}(0)=x_{i 0} \\
u_{i} \in U_{i} \quad \forall i=1 \ldots N
\end{array}\right.}
\end{gathered}
$$

Pour déterminer la solution optimale globale, il suffit en effet de résoudre $N$ problèmes partiels indépendants du type :

$$
\begin{gathered}
\underset{\operatorname{Max}_{i} \in U_{i}}{\operatorname{ax}}\left[\int_{0}^{T} L_{i}\left(x_{i}, u_{i}, t\right) \mathrm{d} t+\Phi_{i}[x(T), T]\right] \\
\stackrel{\circ}{x}_{i}=f_{i}\left(x_{i}, t, u_{i}\right) \quad x_{i}(0)=x_{i 0}
\end{gathered}
$$

- L'organisation tendue vers un même but constitue elle aussi un cas particulier extrêmement intéressant. Lorsque l'organisation possède une fonction d'évaluation additive et que les décisions de chacun de ses membres ont elles-mêmes des effets additifs, on peut envisager un schéma décentralisé. En effet le problème global

$$
\underset{\left[u_{1}, u_{i}, u_{N}\right]}{\operatorname{Max}}\left[\int_{0}^{T} \sum_{i=1}^{N} L_{i}\left(x_{i}, u_{i}, t\right) \mathrm{d} t+\sum_{i=1}^{N} \Phi_{i}[x(T), T]\right]
$$

sous les contraintes

$$
\mid \begin{aligned}
& u_{i} \in U_{i} \\
& \stackrel{\circ}{x}=\sum_{i=1}^{N} f_{i}\left(x_{i}, u_{i}, t\right)
\end{aligned}
$$

se décompose en $N$ optimisations partielles de la forme :

$$
\operatorname{Max} \int_{0}^{T} L_{i}\left(x_{i}, u_{i}, t\right) t+\Phi_{i}[x(T), T]
$$

sous les contraintes

$$
\mid \begin{aligned}
& u_{i} \in U_{i} \\
& \stackrel{\circ}{x}=\sum_{i=1}^{N} f_{i}\left(x_{i}, u_{i}, t\right)
\end{aligned}
$$

Pour ce faire, il suffit que chaque organe de décision $(i)$ connaisse l'ensemble du vecteur $\psi$, c'est-à-dire l'ensemble des prix d'usage. Mais ces prix d'usage communs à l'ensemble de l'organisation sont l'objet d'un calcul décentralisé. En effet, les composantes $\psi_{i}$ du vecteur $\psi$ relatives aux variables d'état $x_{i}$ sont données par l'équation différentielle :

$$
\stackrel{\wp}{\psi}_{i}=-\nabla x_{i}\left[L_{i}\left(x_{i}, u_{i}, t\right)+\psi_{i} f_{i}\left(x_{i}, u_{i}, t\right)\right]
$$


Elles peuvent donc être calculées par l'organe $(i)$. Toutefois pour que les optimisations dynamiques partielles coïncident avec l'optimisation dynamique globale, il faut que chaque centre informe ses partenaires des prix d'usage qu'il vient d'élaborer et des décisions correspondantes.

- L'organisation hiérarchisée est une structure décisionnelle dans laquelle on peut ordonner les différents organes de telle sorte que chaque centre peut être influencé par les centres situés au-dessus de lui, mais ne subit aucune influence des centres situés aux niveaux inférieurs. Cette définition signifie que le centre (1) doit résoudre un problème de commande optimale

$$
\underset{\boldsymbol{u}_{1} \in U_{1}}{\operatorname{Max}}\left[J_{1}=\int_{0}^{T} L_{1}\left(x_{1}, u_{1}, t\right) d t+\Phi_{1}[x(T), T]\right]
$$

sous les contraintes $\mid \stackrel{\circ}{x}_{1}=f_{1}\left(x_{1}, u_{1}, t\right) ; \quad x_{1}(0)=x_{10}$

tandis que le centre (2) se trouve quant à lui confronté à un problème du type :

$$
\operatorname{Max}_{U_{2} \in U_{2}}\left[J_{2}=\int_{0}^{T} L_{2}\left(x_{1}, x_{2}, u_{1}, u_{2}, t\right) \mathrm{d} t+\Phi_{2}[x(T), T]\right]
$$

sous les contraintes $\stackrel{\circ}{x_{2}}=f_{2}\left(x_{1}, x_{2}, u_{1}, u_{2}, t\right) ; \quad x_{2}(0)=x_{20}$

Il est clair que l'étude d'une structure hiérarchique de ce type conduit à résoudre une suite de problèmes de commande optimale.

Enfin il va de soi que toutes les structures d'organisations poisines de ces schémas particuliers (jeux différentiels à somme presque nulle, organisations faiblement couplées ou imparfaitement décomposables, organisations à buts presque identiques organisations incomplètement hiérarchisées) se prêtent elles aussi au calcul dans la mesure où le développement en série de Taylor des écarts entre les deux formalisations peut être limité aux termes du second ordre [9].

\subsection{Exemples}

Les situations conflictuelles ou coopératives sont si nombreuses qu'il paraît à première vue inutile de donner des exemples économiques de jeux différentiels et ceci d'autant plus que les possibilités pratiques de calcul restent à l'heure actuelle relativement limitées. Cependant le simple fait de construire un modèle est toujours un exercice extrêmement intéressant car toute formalisation est à la fois une source de questions et une puissante incitation à s'engager dans la recherche d'une solution.

Nous nous bornerons ici à préciser sur un exemple les relations qui existent entre prix d'échange et prix d'usage puis à montrer l'intérêt des jeux différentiels dans l'élaboration de la stratégie commerciale de la firme. 


\subsubsection{Relation entre prix d'usage et prix d'échange}

On ne doit pas confondre les prix d'usage que les différents joueurs attribuent aux stocks de ressources qu'ils détiennent et le prix auquel ils effectuent leurs transactions. Toutefois les prix d'usage sont calculés à partir des prix d'échanges tandis que ces derniers s'établissent de telle sorte que sur la trajectoire d'équilibre les quantités vendues coïncident avec les quantités achetées.

Pour préciser ces affirmations considérons deux entreprises concurrentes (1) et (3) qui s'approvisionnent chez le même fournisseur et intéressons-nous aux stocks de marchandises détenus par chacune des trois entreprises : ces stocks sont représentés par trois vecteurs $x_{1} x_{2} x_{3}$.

Désignons par :

$u_{1} u_{2}, u_{3}$ les décisions de production de chacune des entreprises au cours de la période $(t, t+d t)$

$u_{12}$ les quantités vendues par l'entreprise (1) à l'entreprise (2)
$\begin{array}{lllll}u_{13} & - & - & - & \text { (1) }\end{array}-$ - (3)

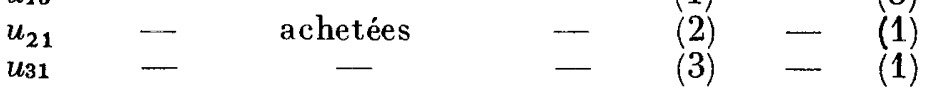

Il est clair que la transaction impose que $u_{12}=u_{21}, u_{13}=u_{31}$ et que ces quantités soient positives.

Soit :

$p_{12}(t)$ le prix d'échange entre (1) et $(2), p_{13}(t)$ le prix d'échange entre (1) et (3)

$b_{1}\left(x_{1}, u_{1}, u_{12}, u_{13}\right)=p_{12} u_{12}+p_{13} u_{13}-\gamma\left(u_{1}, x_{1}\right)$ le bénéfice réalisé par l'entreprise (1) au cours de la période $(t, t+d t)$

$\beta_{2}\left(x_{2}, u_{2}, x_{3}, u_{3}, u_{21}\right)$ et $\beta_{3}\left(x_{3}, u_{3}, x_{2}, u_{2}\right)$ les fonctions d'évaluation respectives des entreprises (2) et (3) au cours de la même période ; ces fonctions dépendent de l'importance de l'entreprise concurrente.

Les équations d'évolution du système s'écrivent :

$$
\begin{aligned}
& \stackrel{\circ}{x}_{1}=x_{1}+u_{1}-u_{12}-u_{13} \\
& \stackrel{\circ}{x}_{2}=x_{2}-g_{2}\left(u_{2}\right)+u_{21} \\
& \stackrel{\circ}{x}_{3}=x_{3}-g_{3}\left(u_{3}\right)+u_{31}
\end{aligned}
$$

$g_{2}\left(u_{2}\right)$ et $g_{3}\left(u_{3}\right)$ étant les quantités de marchandises nécessaires pour produire $u_{2}$ ou $u_{3}$

Pour un système de prix d'échange $\left(p_{12}, p_{1^{3}}\right)$ les conditions nécessaires qui caractérisent la trajectoire d'équilibre s'écrivent :

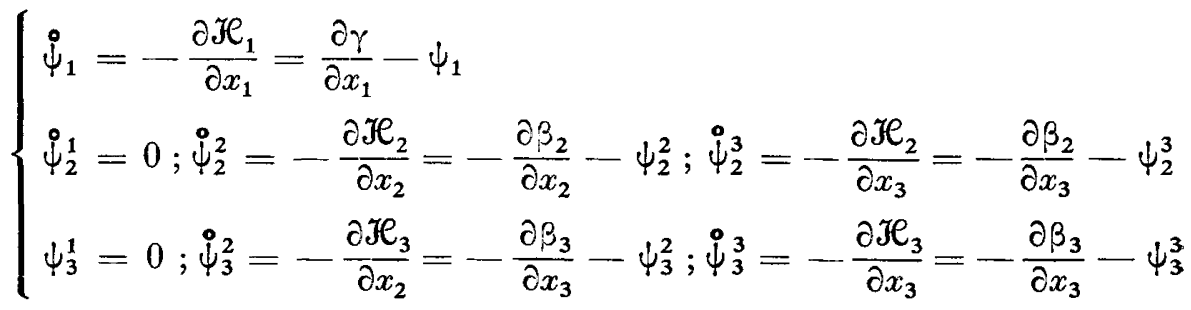




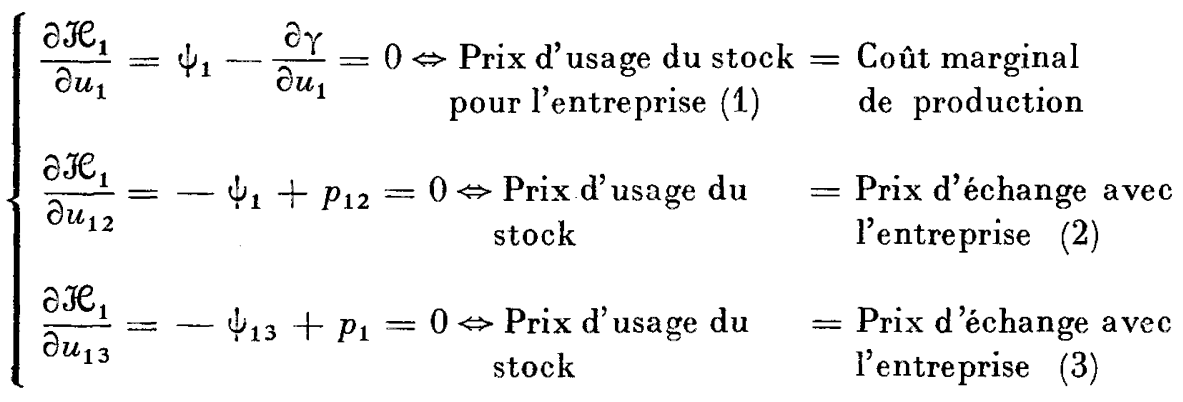

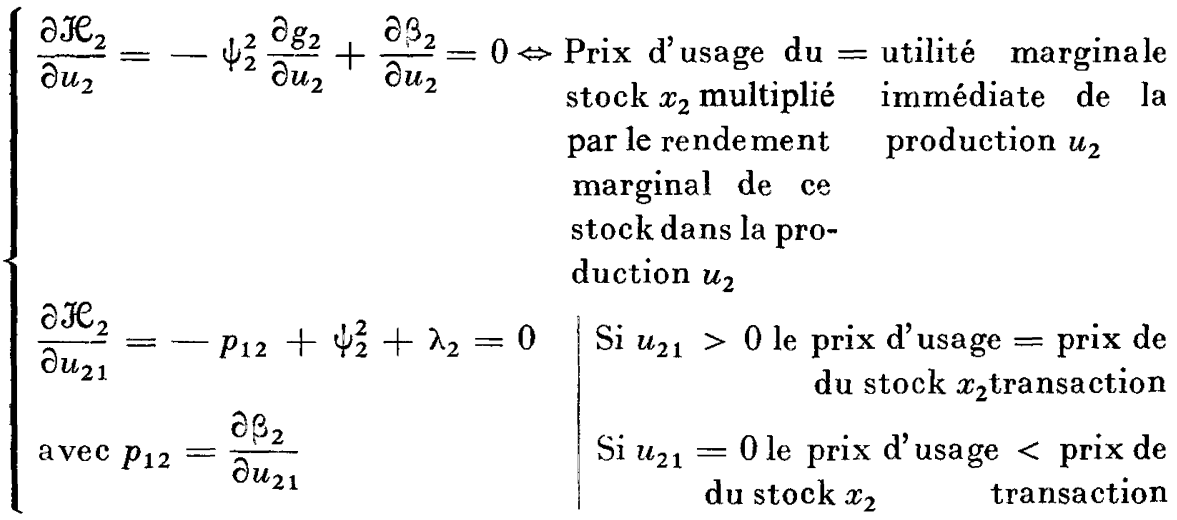

idem pour l'entreprise 3

Si donc il existe une transaction effective entre deux entreprises, c'est que le prix d'usage des stocks de marchandises est identique pour les deux parties et égal au prix de transaction.

Mais parmi ces trajectoires d'équilibre, on ne doit retenir que celles qui vérifient à tout instant la double égalité $u_{21}=u_{12} ; u_{3_{1}}=u_{1^{3}}$ ce qui conduit à comparer le coût marginal de production de l'entreprise (1) et les utilités marginales des entreprises (2) et (3). Or rien ne nous autorise à écrire que pour un prix de transaction donné $p=p_{13}=p_{12}$, la double égalité se trouve vérifiée. On peut cependant penser qu'il existe (1) un ou plusieurs prix de transaction $p^{*}(t)$ pour lesquels on vérifie simultanément :

$$
\mid \begin{aligned}
& u_{21}=u_{12} ; u_{31}=u_{13} \\
& p^{*}=\frac{\partial \gamma}{u_{1}}=\psi_{1}=\psi_{2}^{2}=\psi_{3}^{3}
\end{aligned}
$$

(1) A notre connaissance la démonstration de l'existence des prix $p^{\star}(t)$ dans le cas général n'a pas encore été faite. Mais, nous disposons, depuis les travaux de G. Debreu en 1954 sur la théorie de la valeur, d'un théorème d'existence dans le cas particulier d'une économie d'échange où il n'y aurait pas d'effets externes. 
La trajectoire correspondante est alors appelée trajectoire d'équilibre effective.

Le lecteur notera que ce schéma diffère sensiblement du modèle classique de l'échange dans lequel par définition il n'existe aucun recouorement du champ d'appréciation des agents économiques (hypothèses des Robinson Crusoé). Ceci est particulièrement sensible si l'on suppose que les trois entreprises forment une coalition. et si l'on cherche les trajectoires maximales effectives de cette coalition On constaterait qu'en raison des structures d'évaluation comparatives des entreprises (2) et (3) les trajectoires d'équilibre ne sont pas des trajectoires maximales.

\subsubsection{Un modèle de stratégie publicitaire}

Dans certains secteurs la compétition entre les entreprises est liée à l'effort publicitaire des différents producteurs. Mais ces efforts ne sont ni totalement concurrents, ni parfaitement complémentaires : c'est pourquoi la théorie des jeux différentiels peut s'avérer quelquefois très utile pour orienter par grande masse l'effort publicitaire d'une entreprise. Nous désignerons par $x$ l'état du marché, c'est-à-dire la quantité de marchandises que le marché est capable d'absorber à une date donnée. L'évolution du marché $\dot{x}$ dépend bien entendu du niveau de consommation $x$, des efforts publicitaires $u_{i}$ des diverses entreprises et aussi de données conjoncturelles (revenu...) que nous représenterons par la variable exogène $t$. D'où l'équation d'évolution :

$$
\stackrel{\circ}{x}=f\left(x, u_{1} \ldots u_{i} \ldots u_{N}, t\right) ; x(0)=x_{0}
$$

On admettra que le but de chaque firme est de choisir la répartition $u_{i}$ de son effort publicitaire entre les différents produits de manière à maximiser son profit à long terme tout en s'imposant d'atteindre à une certaine époque une certaine part du marché. Le profit instantané estévidemment croissant en fonction de l'état du marché et décroissant en fonction des dépenses publicitaires. On obtient donc la formulation classique :

$$
\begin{aligned}
\operatorname{Max} & \int_{0}^{T} L_{i}\left(x, u_{i}, t\right) \mathrm{d} t+\Phi_{i}[x(T), T) \\
& \mid \begin{array}{l}
\stackrel{\circ}{x}=f\left(x, u_{i} \ldots u_{N}, t\right) ; x(0)=x_{0} \\
u_{i} \geqslant 0
\end{array}
\end{aligned}
$$

L'avantage de cette formulation est triple :

1) Elle permet à l'entreprise d'arbitrer entre les dépenses immédiates de publicité et les nouvelles potentialités que ces dépenses permettent de susciter.

2) Elle oblige l'entreprise à distinguer le marché protégé où elle est seule à agir et le marché concurrentiel où ses produits se trouvent confrontés avec les produits similaires des autres entreprises. 
3) Concernant ce dernier marché, la formalisation ci-dessus impose de mesurer très soigneusement les effets de complémentarité et les effets de concurrence.

Ceci étant, la difficulté principale consiste autant à informer le modèle qu'à le traiter.

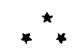

En conclusion, la théorie des jeux différentiels apparaît à l'heure actuelle aux yeux de nombreux spécialistes comme un outil d'analyse particulièrement adapté à l'étude des conflits économiques. Toutefois, à l'exception du jeu linéaire-quadratique ou des formes d'organisations particulières que nous venons d'énumérer, elle appartient encore largement au domaine de la recherche.

Les difficultés principales se situent à plusieurs niveaux :

- Au niveau des méthodes de résolution, la solution des jeux différentiels dépend des études qui sont en cours sur les systèmes d'équations aux dérivées partielles.

- Au niveau de l'information, tout modèle de jeu différentiel contraint les spécialistes à identifier convenablement le fonctionnement du système réel pour pouvoir écrire les équations d'évolution.

- Enfin au niveau de la conceptualisation, il semble que les problèmes posés par la dynamique du jeu coopératif restent non résolus. En effet chacun peut observer qu'au cours de l'évolution les coalitions se font et se défont et que sur ce point la théorie que nous venons d'exposer ne nous apporte aucune réponse.

\section{BIBLIOGRAPHIE}

[1] R. Isaacs, Differential Games, Wiley N. Y. 1965.

[2] J. H. CASE, Equilibrium points of $\mathrm{N}$ person differential games, University of Michigan Dept of Ind. Eng. Tech. Rep., 1967-1.

[3] A. W. Starr et Y. C. Ho, "Non zero-sum differential games ", J. Optimization theory and applications, $3, \mathrm{n}^{\circ} 3$, March 1969.

[4] I. B. Rhodes and D. G. Luenberger, "Differential games with imperfect state information ", IEEE IEEE Tr, AC, 1969.

[5] R. D. BEHN and Y. C. Ho, "On a class of linear stochastic differential games", IEEE Tr, AC 13, no 13, June 1968.

[6] Rockafellar, Convex analysis.

[7] N. O. Da Cunha et E. Polak, "Constrained minimization under vector-valued criteria in finite dimensional spaces ", Memorandum ERLM 188, Oct. 1966, Universisty of California Berkeley.

[8] Y. C. Ho, A. E. Bryson and S. Baron, "Differential games and optimal pursuitevasion strategies, IEEE tr., AC 10, no 4, 1964.

[9] Starr A. W., Non zero-sum differential games concepts and models, juin 1969, tech report No 590 .

[10] Albouy M. Regulation dans l'entreprise, à paraître Dunod 1970. 